\title{
Pacific
}

Journal of

Mathematics

\section{MINIMAL SETS OF PERIODS FOR TORUS MAPS VIA NIELSEN NUMBERS}

Lluís AlsedÀ, Stewart BaLdwin, Jaume Llibre, Richard SWANSON AND WIESLAW SZLENK 
PACIFIC JOURNAL OF MATHEMATICS

Vol. 169, No. 1, 1995

\title{
MINIMAL SETS OF PERIODS FOR TORUS MAPS VIA NIELSEN NUMBERS
}

\author{
L. Alsedì, S. Baldwin, J. Llibre, R. Swanson and \\ W. SZLENK
}

The main results in this paper concern the minimal sets of periods possible in a given homotopy class of torus maps. For maps on the 2 -torus, we provide a complete description of these minimal sets. A number of results on higher dimensional tori are also proved; including criteria for every map in a given homotopy class to have all periods, or all but finitely many periods.

1. Introduction. In dynamical systems, it is often the case that topological information can be used to study qualitative properties of the system. This article deals with the problem of determining the set of periods (of the periodic orbits) of a mapping given the homotopy class of the mapping. To fix terminology, suppose $f$ is a continuous self-map on the manifold $M$. A fixed point of $f$ is a point $x$ in $M$ such that $f(x)=x$. We will call $x$ a periodic point of period $n$ if $x$ is a fixed point of $f^{n}$ but is not fixed by any $f^{k}$, for $1 \leq k<n$.

Denote by $\operatorname{Per}(f)$ the set of natural numbers corresponding to periods of periodic orbits of $f$.

Even for circle maps $f: \mathbf{S}^{1} \rightarrow \mathbf{S}^{1}$ the relation between the degree of $f$ and the set $\operatorname{Per}(f)$ is interesting and nontrivial (see [5], [3] and, for more details, $[\mathbf{1}])$. Let $\mathbb{N}, \mathbb{Z}$, and $\mathbb{R}$ denote, respectively the natural numbers, integers, and reals. Suppose that $f$ is a circle map of degree $d$. Then we have

(1) For $d=1, f$ may have no periodic points.

(2) For $d \geq 2$ or $d \leq-3, \operatorname{Per}(f)=\mathbb{N}$.

(3) For $d=0$ or $d=-1, f$ has a fixed point.

(4) For $d=-2, \operatorname{Per}(f) \supset \mathbb{N} \backslash\{2\}$.

One of the objects of this paper is to study the set $\operatorname{Per}(f)$ for continuous self-maps defined on the $m$-torus $\mathbb{T}^{m}=\mathbf{S}^{1} \times \stackrel{(m)}{\cdots} \times \mathbf{S}^{1}$. 
Our aim is to provide a description of the minimal set of periods (see below) attained within the homotopy class of a given torus map $f: \mathbb{T}^{m} \rightarrow \mathbb{T}^{m}$. We also present a few results, described below, in a more general setting.

Toward this end, it is convenient to distinguish among several subsets of the natural numbers $\mathbf{N}$. First, there is the set of periods, $\operatorname{Per}(f)$, mentioned above. When the mapping $g: M \rightarrow M$ is homotopic to $f$, we shall write $g \simeq f$. Define the minimal set of periods of $f$ to be the set

$$
\operatorname{MPer}(f)=\bigcap_{g \simeq f} \operatorname{Per}(g)
$$

In order to determine $\operatorname{MPer}(f)$, one can use Nielsen fixed point theory (see Sections 2 and 3 for details). To each map $f: M \rightarrow$ $M$, where $M$ is a compact "Euclidean neighborhood retract", we can assign the Nielsen number $N(f)$. This number is a homotopy invariant such that

$$
N(f) \leq \#\{x: f(x)=x\} .
$$

In Section 4 , in the case of torus maps $f: \mathbb{T}^{m} \rightarrow \mathbb{T}^{m}$, we estimate the sets $\operatorname{MPer}(f)$ in terms of the numbers $N\left(f^{k}\right), k \geq 1$. To make this clear, let $f_{* 1}: H_{1}\left(\mathbb{T}^{m}, \mathbb{Z}\right) \rightarrow H_{1}\left(\mathbb{T}^{m}, \mathbb{Z}\right)$ denote the first induced homology map, corresponding to some $m \times m$ matrix $A$ of integers. Then the linear map $A: \mathbb{R}^{m} \rightarrow \mathbb{R}^{m}$ covers a unique algebraic endomorphism $f_{A}: \mathbb{T}^{m} \rightarrow \mathbb{T}^{m}$ with $f_{A} \simeq f$. In Section 3 we show (Proposition 3.4) that

$$
\operatorname{MPer}(f)=\operatorname{Per}\left(f_{A}\right) \backslash\left\{k \in \mathbf{N}: N\left(f_{A}^{k}\right)=0\right\} .
$$

Moreover, if no eigenvalue of $A$ is a root of unity then $\operatorname{MPer}(f)=$ $\operatorname{Per}\left(f_{A}\right)$.

In fact, the above equality was conjectured by Halpern [6], who proved the result in the case of all eigenvalues equal to roots of unity.

One of the goals of this paper is to show in detail how $\operatorname{MPer}(f)$ can be determined solely from the knowledge of the sequence of Nielsen numbers $\left\{N\left(f^{k}\right)\right\}_{k \geq 1}$. Thus, when the Nielsen number is easily computable, it provides a good tool for investigating the set of periods common to a given homotopy class. This approach was used by Halpern [6] for torus maps. 
To compute Nielsen numbers on the $m$-torus, we proceed as follows. Given a map $f$, the Nielsen number of $f$ equals the absolute value of the Lefschetz number: $N(f)=|L(f)|$ by a theorem of Brooks, Brown, Pak and Taylor [4]. Recall that the Lefschetz number is given by

$$
L(f)=\sum_{k=0}^{m}(-1)^{k} \operatorname{Trace}\left(f_{* k}\right),
$$

where $f_{* k}: H_{k}\left(\mathbb{T}^{m}, \mathbb{Z}\right) \rightarrow H_{k}\left(\mathbb{T}^{m}, \mathbb{Z}\right)$ is the $k^{\text {th }}$ order homology homomorphism of $f$. With $A$ as above, one can show

$$
L(f)=L\left(f_{A}\right)=\operatorname{det}(I-A) .
$$

Thus, for each $k \geq 1$

$$
N\left(f^{k}\right)=\left|\operatorname{det}\left(I-A^{k}\right)\right|,
$$

and if $N\left(f^{k}\right) \neq 0$, the mapping $f_{A}^{k}: \mathbb{T}^{m} \rightarrow \mathbb{T}^{m}$ has exactly the number of fixed points given by $N\left(f^{k}\right)$ (see Lemma 2 of [6]).

We now describe the main results of each section (2-6) of this article. In Section 2, we obtain results on the set of periods of a map $f$ from a compact Euclidean neighborhood retract into itself by studying the behavior of the sequence $\left\{N\left(f^{k}\right)\right\}_{k \geq 1}$. In Sections 3 and 4 , we specialize to torus maps $f: \mathbb{T}^{m} \rightarrow \mathbb{T}^{m}$. There we prove that if the sequence of Nielsen numbers $\left\{N\left(f^{k}\right)\right\}_{k \geq 1}$ is strictly increasing, then $\operatorname{Per}(f)=\mathbb{N}$ (Theorem 4.8). In addition we show that if the homology map $f_{* 1}$ has an eigenvalue of modulus greater than one and no eigenvalue equal to a root of unity, then $\operatorname{Per}(f)$ is cofinite in $\mathbf{N}$ (Corollary 4.2). A useful tool for determining the set $\operatorname{Per}(f)$ in many cases is the following result (Theorem 4.14):

TheOREM A. If $f: \mathbb{T}^{m} \rightarrow \mathbb{T}^{m}$ is continuous, $\tau>1$, and $N\left(f^{n+1}\right) / N\left(f^{n}\right)>\tau$ for all $n \geq n_{0}$, then $\operatorname{Per}(f)$ contains all integers greater than or equal to the maximum of $2 n_{0}$ and $4\left(1+\left|\frac{\log (\tau-1)}{\log \tau}\right|\right)$.

The most general result of Section 4 (Corollary 4.3) can be summarized as follows:

TheOREM B. Given any torus map $f: \mathbb{T}^{m} \rightarrow \mathbb{T}^{m}$ then one of the following must occur:

(1) All eigenvalues of $f_{* 1}$ are equal to zero or a root of unity and, hence, the sequence of Nielsen numbers $\left\{N\left(f^{k}\right)\right\}_{k \geq 1}$ is periodic. 
(2) There exists a cofinite subset $C \subseteq \mathbb{N}$ such that $\operatorname{Per}(f) \supset C \backslash$ $\left\{n: N\left(f^{n}\right)=0\right\}$.

Except for the trivial case when 1 is an eigenvalue of $f_{* 1}$, exactly one of the above statements occurs.

At the end of Section 4, we completely describe all the possibilities for the sets $\operatorname{MPer}(f)$ in dimension 2. In this fashion, we obtain results much in the spirit of those cited above for circle maps. The role of the degree is now played by the trace $t$ and determinant $d$ of the first homology homomorphism $f_{* 1}: H_{1}\left(\mathbb{T}^{2}, \mathbb{Z}\right) \rightarrow H_{1}\left(\mathbb{T}^{2}, \mathbb{Z}\right)$. When $d=0$, the situation reduces to the circle case. We can describe our results entirely in terms of the "position" of $f_{* 1}$ in the $(t, d)$ coordinate plane. The periodic structure, up to homotopy, is determined by four key lines and nine exceptional cases. The exceptional cases are:

$$
\begin{array}{r}
(t, d) \in\{(-2,1),(-2,2),(-1,0),(-1,1), \\
(-1,2),(0,0),(0,1),(0,2),(1,1)\}
\end{array}
$$

and are analyzed in the table at the end of Section 4.

With the exception of those 9 specific values, we can conclude the following

(1) If $t-d=1$, then $\operatorname{MPer}(f)=\emptyset$.

(2) If $t \neq 0$ and $t+d=-1$, then $\operatorname{MPer}(f)=\{n: n$ is odd $\}$.

(3) If $t+d=0$ or $t+d=-2$, then $\operatorname{MPer}(f)=\mathbf{N} \backslash\{2\}$.

(4) If $(t, d)$ does not lie on one of the four lines in $(1)-(3)$, then $\operatorname{Per}(f)=\mathbf{N}$.

2. Periods and Nielsen numbers. The goal of this section is to obtain sufficient conditions to determine if a given period occurs for a continuous map in terms of its Nielsen numbers.

The Nielsen fixed point theory is defined for continuous self-maps of Euclidean neighborhood retracts (ENR). These are spaces which can be embedded in some $\mathbb{R}^{n}$ in such a way that the image of the embedding is a retraction of a neighborhood to itself.

Let $f: E \rightarrow E$ be a continuous map of a compact ENR. The Nielsen number $N(f)$ of $f$ is defined as follows. First an equivalence relation $\sim$ is defined on the set $F$ of fixed points of $f$. Two fixed points $x, y$ are equivalent, $x \sim y$, provided there is a path $\gamma$ in 
$E$ from $x$ to $y$ such that $f \circ \gamma$ and $\gamma$ are homotopic and the homotopy fixes endpoints. The set of equivalence classes $F / \sim$ is known to be finite and each equivalence class is compact. Each of these equivalence classes will be called a fixed point class. Using a fixed point index, we may assign an index $i_{f}(C)$ to each fixed point class $C$. An essential class is a fixed point class $C$ such that $i_{f}(C) \neq 0$. Then the Nielsen number is the number of essential classes. For more details on the Nielsen number see [8] or [9].

Notice that from the definition of Nielsen number, $f$ has at least $N(f)$ fixed points. The main difference between the Lefschetz and the Nielsen numbers is that, in general, the Lefschetz number only gives existence of fixed points, while the Nielsen number provides a lower bound for the number of fixed points.

The following result explains the importance of the Nielsen number in fixed point theory. If $f: E \rightarrow E$ is a continuous map of a compact ENR, then each map $g: E \rightarrow E$ homotopic to $f$ has at least $N(f)$ fixed points. Furthermore, $N(f)=N(g)$.

Until further notice (i.e. after the proof of Theorem 2.12), $f$ will be a continuous self-map of a compact ENR. Also any divisor of $n \in \mathbb{N}$ different from $n$ will be called a proper divisor of $n$.

As it is well known, a fixed point of $f^{n}$ need not have period $n$, so it is often useful to have a method for telling the difference between "real" and "false" periodic points of period $n$ (i.e., points having period some proper divisor of $n$ ). Given $f$, let $\operatorname{NFP}(f)$ be the number of fixed points of $f$. Then, if for example $\operatorname{NFP}\left(f^{6}\right)$ is finite, it is easy to see that $f$ has a periodic point of period 6 if and only if $\operatorname{NFP}\left(f^{6}\right)>\operatorname{NFP}\left(f^{3}\right)+\operatorname{NFP}\left(f^{2}\right)-\operatorname{NFP}(f)$. One of the main goals of this section is to get similar results when "NFP" is replaced by " $N$ ". Since we only know that $N\left(f^{n}\right) \leq \operatorname{NFP}\left(f^{n}\right)$, this replacement is not a trivial one. As we only have an inequality, we concentrate on sufficient conditions for $f$ to have a periodic point of period $n$. This is the motivation for the next lemma and the index assumption below.

LEMMA 2.1. Each fixed point class of $f^{k}$ is contained in a fixed point class of $f^{n}$ if $k$ divides $n$.

Proof. Let $x$ and $y$ be points of a fixed point class of $f^{k}$. Then there is a path $\gamma$ from $x$ to $y$ such that $f^{k} \circ \gamma$ and $\gamma$ are homotopic 
by $H$ and $H$ fixes endpoints. It is easy to see that the paths $f^{n} \circ \gamma$ and $f^{n-k} \circ \gamma$ from $x$ to $y$ are homotopic by $f^{n-k} \circ H$, and $f^{n-k} \circ H$ fixes endpoints.

The following assumption, which we call the "index assumption", is somewhat restrictive but it is a simple property, true on the torus, which assures the validity of the results of this section. Also, as pointed out to us by B. Jiang, the index assumption is true for all orientation preserving homeomorphisms of surfaces.

INDEX ASSUMPTION FOR $f$. For each $k, r \in \mathbb{N}$ let $C$ be a fixed point class of $f^{k}$ and let $\bar{C}$ be the fixed point class of $f^{k r}$ which contains $C$. If $\bar{C}$ is essential then $C$ is essential.

In the rest of the section we assume that our map $f$ satisfies the index assumption.

The next result gives sufficient conditions to assure that $n \in$ $\operatorname{Per}(f)$ in terms of the Nielsen numbers of the iterates of $f$.

Proposition 2.2. Assume that

$$
N\left(f^{n}\right)>\sum_{\frac{n}{k} \text { prime }} N\left(f^{k}\right)
$$

Then $f$ has a periodic point of period $n$.

Proof. Let $r=N\left(f^{n}\right)$ and let $C_{1}, C_{2}, \ldots C_{r}$ be the essential classes of $f^{n}$. For each $j, 1 \leq j \leq r$, pick $x_{j} \in C_{j}$. If some $x_{j}$ has period $n$, then we are done. So assume no $x_{j}$ has period $n$. Then, for each $j$, there is a $k_{j}$ so that $n / k_{j}$ is prime and $f^{k_{j}}\left(x_{j}\right)=x_{j}$. For each $j$, the fixed point class of $x_{j}$ for $f^{k_{j}}, \widetilde{C}_{j}$, is contained in $C_{j}$ (see Lemma 2.1). But the index assumption implies that $\widetilde{C}_{j}$ is an essential class of $f^{k_{j}}$. This contradicts

$$
N\left(f^{n}\right)>\sum_{\frac{n}{k} \text { prime }} N\left(f^{k}\right)
$$

(since the $C_{j}$ 's are pairwise disjoint).

For each $n \geq 2$, we define $\alpha_{n}$ to be the unique root of the polynomial $x^{n}-\sum_{\frac{n}{k}} x_{\text {prime }} x^{k}$ which lies in $[1,2)$. 
REMARK 2.3. If $n$ is a prime or a power of a prime, then $\alpha_{n}=1$ (since there is exactly one $k$ such that $\frac{n}{k}$ is prime).

LEMMA 2.4. If $N\left(f^{n}\right)>\alpha_{n}^{n-k} N\left(f^{k}\right)$ for each $\frac{n}{k}$ prime, then $f$ has a periodic point of period $n$.

Proof. Since

$$
\begin{aligned}
N\left(f^{n}\right) & =\left(\frac{1}{\alpha_{n}^{n}} \sum_{\frac{n}{k} \text { prime }} \alpha_{n}^{k}\right) N\left(f^{n}\right)=\frac{1}{\alpha_{n}^{n}} \sum_{\frac{n}{k} \text { prime }} \alpha_{n}^{k} N\left(f^{n}\right) \\
& =\sum_{\frac{n}{k} \text { prime }} \alpha_{n}^{-(n-k)} N\left(f^{n}\right)>\sum_{\frac{n}{k} \text { prime }} N\left(f^{k}\right),
\end{aligned}
$$

from Proposition 2.2 we are done.

Next we shall obtain sufficient conditions in order that a set of the form $\left\{n \in \mathbb{N}: n \geq n_{0}\right\}$ be contained in $\operatorname{Per}(f)$.

For each $\tau, 1<\tau<2$, and each integer $j \geq 1$, we define $\gamma_{\tau}(j)$ to be the least integer $\gamma \geq 1$ such that $\tau^{\gamma} \geq \sum_{i=0}^{j-1} \tau^{i}$. We also define $\Gamma_{\tau}(j)$ by the formula $\left[\log \left(\tau^{j}-1\right)-\log (\tau-1)\right] / \log \tau$. Since $\Gamma_{\tau}(j)$ is just the result of solving the equation $\tau^{\gamma}=\left(\tau^{j}-1\right) /(\tau-1)$ for $\gamma$, it is easy to see that $\gamma_{\tau}(j)$ is always the least positive integer greater than or equal to $\Gamma_{\tau}(j)$. Moreover, $\Gamma_{\tau}(j)-j$ is an increasing function of $j$ and $\lim _{j \rightarrow \infty}\left(\Gamma_{\tau}(j)-j\right)=-\log (\tau-1) / \log \tau$. Thus, for each such $\tau$ there is an integer $n_{\tau}$ such that $\gamma_{\tau}(j) \leq j+n_{\tau}$ for all $j$.

In what follows $\kappa$ will denote $\alpha_{6}=1.22074408 \ldots$, i.e. the root of the polynomial $x^{4}-x-1$ which is contained in $(1,2)$. The next two results show the basic properties of the number $\kappa$.

LEMma 2.5. We have $\gamma_{\kappa}(1)=1, \gamma_{\kappa}(2)=4, \gamma_{\kappa}(3)=7, \gamma_{\kappa}(4)=$ $9, \gamma_{\kappa}(i)=i+6$ for $i=5,6, \gamma_{\kappa}(i)=i+7$ for $i=7,8,9,10,11$ and $\gamma_{\kappa}(i)=i+8$ for $i \geq 12$.

Proof. The first twelve values of $\gamma_{\kappa}$ are obtained by direct computation. Since $\Gamma_{\kappa}(j)-j$ is an increasing function of $j$ and converges to $-\log (\kappa-1) / \log \kappa<8$, the lemma follows.

As usual, $[x]$ denotes the integer part of $x \in \mathbb{R}$. 
LEMMA 2.6. For any positive integer $n, \kappa \geq \alpha_{n}$.

Proof. Since $\gamma_{\kappa}(j) \leq j+8$ for all $j \geq 1$ (see Lemma 2.5), we have that $\gamma_{\kappa}(n-8) \leq n$ and $n-9 \geq\left[\frac{n}{2}\right]$ for all $n \geq 18$. Therefore,

$$
\kappa^{n} \geq \kappa^{\gamma_{\kappa}(n-8)} \geq \sum_{i=0}^{n-9} \kappa^{i} \geq \sum_{i=0}^{[n / 2]} \kappa^{i}>\sum_{\frac{n}{2} \text { prime }} \kappa^{i}
$$

Thus, $\kappa>\alpha_{n}$ for $n \geq 18$.

If $n \in\{1,2,3,4,5,7,8,9,11,13,16,17\}$ then $\alpha_{n}=1$ and if $n \in$ $\{10,12,14,15\}$ then it follows that $\kappa \geq \alpha_{n}$ by direct computation.

THEOREM 2.7. If the ratio $N\left(f^{n+1}\right) / N\left(f^{n}\right)$ is well-defined and greater than or equal to $\kappa$ for all $n \geq 1$, then $\operatorname{Per}(f)=\mathbb{N}$.

Proof. In view of Lemma 2.6, for $n \geq 2$ we have

$$
N\left(f^{n}\right)>\kappa^{n-k} N\left(f^{k}\right) \geq \alpha_{n}^{n-k} N\left(f^{k}\right) .
$$

Then the theorem follows from Lemma 2.4.

We note that since $\kappa$ is irrational, the hypothesis $N\left(f^{n+1}\right) / N\left(f^{n}\right)$ $\geq \kappa$ is equivalent to $N\left(f^{n+1}\right) / N\left(f^{n}\right)>\kappa$.

We shall prove that the constant $\kappa$ appearing in the assumptions of Theorem 2.7, in some sense, is the best possible in order that $\operatorname{Per}(f)=\mathbb{N}$ (see Theorem 4.15). Because the result depends on information concerning tori, we postpone it until after we have investigated maps on these spaces in Section 4.

Now we are interested in studying what can be said about the set of periods if in Theorem 2.7 we replace the assumption $n \geq 1$ by $n \geq n_{0}$ for some $n_{0}>1$. To do this we need the following result. For each $n \in \mathbb{N}$ we denote by $\xi(n)$ the number of distinct prime factors of $n$.

LEMma 2.8. Let $n \geq 2$ be a positive integer and let $k$ be the greatest proper divisor of $n$. Then $n-\gamma_{\kappa}(\xi(n))+\xi(n)-1 \geq k$

Proof. From Lemma 2.5 we have $\gamma_{\kappa}(\xi(n))-\xi(n) \leq 8$. Then $n-\gamma_{\kappa}(\xi(n))+\xi(n)-1 \geq n-9 \geq n / 2 \geq k$ if $n \geq 18$. The 
remaining cases $(n<18)$ are easily checked because $\xi(n) \leq 2$ and $\gamma_{\kappa}(\xi(n)) \in\{1,4\}$.

THEOREM 2.9. Suppose that the ratio $N\left(f^{n+1}\right) / N\left(f^{n}\right)$ is welldefined and greater than $\kappa$ for all $n \geq n_{0}$, and $N\left(f^{n}\right) \leq N\left(f^{n_{0}}\right)$ for all $n \leq n_{0}$. Then $f$ has periodic points of all periods $n$ such that $n \geq n_{0}+\gamma_{\kappa}(\xi(n))$.

Proof. Suppose $n \geq n_{0}+\gamma_{\kappa}(\xi(n))$. Note that if $i>k \geq n_{0}$, then $N\left(f^{i}\right)>\kappa^{i-k} N\left(f^{k}\right)$, and if $i \geq n_{0}$ and $j \leq n_{0}$ then $N\left(f^{i}\right) \geq N\left(f^{j}\right)$. Set $j=\xi(n)$ and $\gamma=\gamma_{\kappa}(j)$. Then

$$
\begin{aligned}
N\left(f^{n}\right) & \geq\left(\frac{1}{\kappa^{\gamma}} \sum_{i=0}^{j-1} \kappa^{i}\right) N\left(f^{n}\right)=\left(\frac{1}{\kappa^{n}} \sum_{i=0}^{j-1} \kappa^{n-\gamma+i}\right) N\left(f^{n}\right) \\
& >\frac{1}{\kappa^{n}} \sum_{i=0}^{j-1} \kappa^{n-\gamma+i}\left(\kappa^{\gamma-i} N\left(f^{n-\gamma+i}\right)\right)=\sum_{i=0}^{j-1} N\left(f^{n-\gamma+i}\right)
\end{aligned}
$$

because $n-\gamma \geq n_{0}$. Since the number of $k$ 's such that $\frac{n}{k}$ is prime is $j$, from Lemma 2.8 it follows

$$
\sum_{i=0}^{j-1} N\left(f^{n-\gamma+i}\right) \geq \sum_{\frac{n}{k} \text { prime }} N\left(f^{k}\right),
$$

and from Proposition 2.2 the result follows.

From Theorem 2.9 we obtain the following characterization of the set $\operatorname{Per}(f)$ for small values of $n_{0}$. Note that in particular we get a new proof of Theorem 2.7.

Theorem 2.10. Suppose that the ratio $N\left(f^{n+1}\right) / N\left(f^{n}\right)$ is welldefined and greater than or equal to $\kappa$ for all $n \geq n_{0}$. Then, the following statements hold.

(1) If $n_{0}=1$, then $\operatorname{Per}(f)=\mathbb{N}$.

(2) If $n_{0}=2$, then $\operatorname{Per}(f) \supset \mathbb{N} \backslash\{2\}$.

(3) If $n_{0} \in\{3,4\}$, then $\operatorname{Per}(f) \supset \mathbb{N} \backslash\{2,3,4,6\}$.

(4) If $n_{0} \in\{5,6\}$, then $\operatorname{Per}(f) \supset \mathbb{N} \backslash\{2,3,4,5,6,8,10\}$.

Proof. For each $n_{0} \geq 1$ let $\widetilde{n}=\widetilde{n}\left(n_{0}\right)$ be the smallest integer $k$ such that $n_{0} \leq k$ and there is a multiple of each $i<n_{0}$ in $\left[n_{0}, k\right]$ (if $n_{0}=1$ 
we set $\widetilde{n}=1)$. Clearly, $\widetilde{n} \leq 2 n_{0}$. We claim that $N\left(f^{n}\right) \leq N\left(f^{\tilde{n}}\right)$ if $n \leq \tilde{n}$.

To prove the claim suppose first that $n_{0} \leq n \leq \tilde{n}$. From the hypothesis, $N\left(f^{\tilde{n}}\right)>\kappa^{\tilde{n}-n} N\left(f^{n}\right) \geq N\left(f^{n}\right)$. Now, assume $n<n_{0}$. If $k$ is the multiple of $n$ in $\left[n_{0}, \widetilde{n}\right]$ then $N\left(f^{n}\right) \leq N\left(f^{k}\right) \leq N\left(f^{\tilde{n}}\right)$. Hence, the claim is proved.

From the claim and Theorem 2.9 we get that $f$ has periodic points of all periods $n$ such that $n \geq \tilde{n}+\gamma_{\kappa}(\xi(n))$. From Lemma 2.5 we have $\widetilde{n}+\gamma_{\kappa}(\xi(n)) \leq \widetilde{n}+8+\xi(n) \leq \widetilde{n}+8+n / 2$ if $n \geq 2$. Therefore, $n \in \operatorname{Per}(f)$ if $n \geq 2 \tilde{n}+16$. Note that for $n_{0} \in\{2,3,4,5,6\}$ we have $\widetilde{n}\left(n_{0}\right)=2 n_{0}-2$ and hence $\widetilde{n}\left(n_{0}\right) \leq 10$ for $n_{0} \in\{1,2,3,4,5,6\}$. Thus, $n \in \operatorname{Per}(f)$ for all $n \geq 36$ if $n_{0} \in\{1,2,3,4,5,6\}$. If $n \leq 35$ we have $\gamma_{\kappa}(\xi(n)) \in\{1,4,7\}$ because $\xi(n) \leq 3$. Hence, again from Theorem 2.9, $n \in \operatorname{Per}(f)$ if $n \geq \tilde{n}+7$ and $n_{0} \in\{1,2,3,4,5,6\}$. Thus, $n \in \operatorname{Per}(f)$ for all $n \geq 17$ if $n_{0} \in\{1,2,3,4,5,6\}$. If $n \leq 16$ we have $\gamma_{\kappa}(\xi(n)) \in\{1,4\}$ because $\xi(n) \leq 2$. Therefore, $n \in \operatorname{Per}(f)$ if $n \geq \widetilde{n}+4$ and $n_{0} \in\{1,2,3,4,5,6\}$.

Note that if $1<n \leq \widetilde{n}$, then $n<\widetilde{n}+\gamma_{\kappa}(\xi(n))$ and we cannot use Theorem 2.9. So we cannot assure that $\{2,3, \ldots, \tilde{n}\}$ is contained in $\operatorname{Per}(f)$. In short, for a given $n_{0} \in\{1,2,3,4,5,6\}$ we only have to check which are the $n \in \mathbb{N}$ such that $\widetilde{n}+1 \leq n \leq \tilde{n}+3$ and $n \geq \tilde{n}+\gamma_{\kappa}(\xi(n))$. We subdivide the rest of the proof into the following cases.

Case 1: $n_{0}=1$. For $n=2,3,4$ we have $n \geq \widetilde{n}+\gamma_{k}(\xi(n))=2$ because $\gamma_{k}(\xi(n))=1$. So $\operatorname{Per}(f)=\mathbf{N}$.

Case 2: $n_{0}=2$. For $n=3,4,5$ we have $n \geq \widetilde{n}+\gamma_{\kappa}(\xi(n))=3$ because $\gamma_{\kappa}(\xi(n))=1$. So $\operatorname{Per}(f) \supset \mathbb{N} \backslash\{2\}$.

Case 3: $n_{0}=3$. For $n=5,7$ we have $n \geq \tilde{n}+\gamma_{k}(\xi(n))=5$ (and $\left.6<\tilde{n}+\gamma_{\kappa}(\xi(6))=8\right)$. So $\operatorname{Per}(f) \supset \mathbf{N} \backslash\{2,3,4,6\}$.

Case 4: $n_{0}=4$. For $n=7,8,9$ we have $\gamma_{\kappa}(\xi(n))=1$ and, as in the above cases, we obtain $\operatorname{Per}(f) \supset \mathbf{N} \backslash\{2,3,4,5,6\}$. However, since $N(f) \leq N\left(f^{4}\right)<\kappa N\left(f^{4}\right)<N\left(f^{5}\right)$ we get that $5 \in \operatorname{Per}(f)$.

Case 5: $n_{0}=5$. For $n=9,11$ we get $\gamma_{\kappa}(\xi(n))=1$, so $\operatorname{Per}(f) \supset$ $\mathbf{N} \backslash\{2,3,4,5,6,7,8,10\}$. From $N(f) \leq N\left(f^{6}\right)<\kappa N\left(f^{6}\right)<N\left(f^{7}\right)$ it follows that $7 \in \operatorname{Per}(f)$.

Case 6: $n_{0}=6$. In a similar way we get $\operatorname{Per}(f) \supset \mathbf{N} \backslash\{2,3,4,5,6,7$, 
$8,9,10,12\}$. From

$$
\begin{aligned}
N(f) & \leq N\left(f^{6}\right)<\kappa N\left(f^{6}\right)<N\left(f^{7}\right), \\
N\left(f^{3}\right) & \leq N\left(f^{6}\right)<\kappa^{3} N\left(f^{6}\right)<N\left(f^{9}\right), \\
N\left(f^{6}\right) & <\kappa^{6} N\left(f^{12}\right)
\end{aligned}
$$

it follows that $\{7,9,12\} \subset \operatorname{Per}(f)$.

3. Periods and Nielsen numbers for torus maps. In this section we shall prove that the results obtained in Section 2 can be used in the study of the minimal sets of periods for torus maps.

Let $A$ be an $m \times m$ matrix of integers. We shall denote by $f_{A}$ : $\mathbb{T}^{m} \rightarrow \mathbb{T}^{m}$ the map covered by the linear map $A: \mathbb{R}^{m} \rightarrow \mathbb{R}^{m}$. We note that then $A$ is a matrix representative of the induced homology homomorphism $\left(f_{A}\right)_{* 1}: H_{1}\left(\mathbb{T}^{m}, \mathbb{Q}\right) \rightarrow H_{1}\left(\mathbb{T}^{m}, \mathbb{Q}\right)$. Hence, from the introduction we have that $N\left(f_{A}^{n}\right)=\left|\operatorname{det}\left(I-A^{n}\right)\right|$. From this equality it follows immediately.

LEMma 3.1. The Nielsen number $N\left(f_{A}^{n}\right)$ vanishes if and only if 1 is an eigenvalue of $A^{n}$.

The following result is a rewriting of Lemma 2 of $[6]$.

Proposition 3.2. If 1 is not an eigenvalue of $A$, then each fixed point of $f_{A}$ is a distinct essential fixed point class of $f_{A}$.

Since $f_{A}^{k}=f_{A^{k}}$ for each $k \geq 1$, from Lemma 3.1 and Proposition 3.2 we get immediately:

Corollary 3.3. The index assumption holds for $f_{A}$.

Now we consider the situation in which $f$ is a torus map and $A$ is a matrix representative of the induced homology homomorphism $f_{* 1}: H_{1}\left(\mathbb{T}^{m}, \mathbb{Q}\right) \rightarrow H_{1}\left(\mathbb{T}^{m}, \mathbb{Q}\right)$. Of course,$f \simeq f_{A}$. We recall that $\operatorname{MPer}(f)$ was defined to be $\bigcap_{g \simeq f} \operatorname{Per}(g)$. The next result shows how to study the set $\operatorname{MPer}(f)$ by using the set $\operatorname{Per}\left(f_{A}\right)$.

Proposition 3.4. The following equalities hold: $\operatorname{MPer}(f)=$ $\operatorname{Per}\left(f_{A}\right) \backslash\left\{k \in \mathbb{N}: N\left(f_{A}^{k}\right)=0\right\}=\operatorname{Per}\left(f_{A}\right) \backslash\{n \in \mathbb{N}: 1$ is an eigenvalue of $\left.A^{n}\right\}$.

Proof. From Lemma 3.1, we know that $\left\{n \in \mathbb{N}: N\left(f_{A}^{n}\right)=0\right\}=$ $\left\{n \in \mathbb{N}: 1\right.$ is an eigenvalue of $\left.A^{n}\right\}$, which proves the second equality. 
For a torus map $g$ we denote by $P_{n}(g)$ the number of periodic points of period $n$ of $g$. We define $M P_{n}(f)$ by $\min \left\{P_{n}(g): g \simeq f\right\}$. Assume that $n$ is such that 1 is not an eigenvalue of $A^{n}$. From Proposition 2.2(ii), Remark 3.6 and Theorem 3.7 of [7], it follows that

$$
M P_{n}(f) \geq \sum_{\tau \subseteq P(n)}(-1)^{\# \tau} N\left(f^{n: \tau}\right),
$$

where $P(n)$ is the set of all distinct primes which divide $n, \# \tau$ denotes the cardinality of the set $\tau$, and $n: \tau=n\left(\prod_{p \in \tau} p\right)^{-1}$. Furthermore, in [11], given $N$, a map $\tilde{f} \simeq f_{A}$ is constructed, such that

$$
M P_{n}(\tilde{f})=\sum_{\tau \subseteq P(n)}(-1)^{\# \tau} N\left(f_{A}^{n: \tau}\right),
$$

for all $n \leq N$. We note that since the empty set is a subset of $P(n)$, the above sums always include the term $N\left(f^{n}\right)$.

By Proposition 3.2 it follows that

$$
\sum_{\tau \subseteq P(n)}(-1)^{\# \tau} N\left(f_{A}^{n: \tau}\right)=P_{n}\left(f_{A}\right) .
$$

Since the Nielsen numbers are invariant under homotopy we get $M P_{n}(f)=P_{n}\left(f_{A}\right)$. Hence, the proposition is proved.

We note that from the above proposition, in particular, we get that if no eigenvalue of $A$ is a root of unity then $\operatorname{MPer}(f)=\operatorname{Per}\left(f_{A}\right)$.

To study the set $\operatorname{MPer}(f)$ we shall analyze the set $\operatorname{Per}\left(f_{A}\right) \backslash\{n \in$ $\left.\mathbb{N}: N\left(f_{A}^{n}\right)=0\right\}$. This will be done in the next three sections. To develop this study we shall use the results obtained in Section 2 . This will be possible in view of Corollary 3.3.

4. On the set of periods for torus maps. In this section we shall prove some results on the set of periods for the $m$-dimensional torus maps.

Let $f$ be an $m$-dimensional torus map and let $A$ be a matrix representative of the induced homology homomorphism $f_{* 1}$. From now on $A$ will be called a matrix associated to $f$. As usual we denote by $f_{A}$ the torus map covered by the linear map $A: \mathbb{R}^{m} \rightarrow \mathbb{R}^{m}$. Then $f \simeq f_{A}$. Since the Nielsen numbers are invariant under homotopy, we have $N\left(f^{n}\right)=N\left(f_{A}^{n}\right)$. Moreover, from the introduction

$$
N\left(f_{A}^{n}\right)=\left|\operatorname{det}\left(I-A^{n}\right)\right|=\prod_{j=1}^{m}\left|1-\lambda_{j}^{m}\right|,
$$


where $\lambda_{1}, \ldots, \lambda_{m}$ are all the eigenvalues of $A$ (in this sequence the eigenvalues are repeated according their multiplicity). In the rest of the paper the number $\left|\operatorname{det}\left(I-A^{n}\right)\right|$ will also be denoted by $N\left(A^{n}\right)$.

We denote by $T_{A}$ the set $\{n \in \mathbf{N}: 1$ is not an eigenvalue of $\left.A^{n}\right\}=\left\{n \in \mathbb{N}: N\left(A^{n}\right) \neq 0\right\}$ (see Lemma 3.1). The main result of this section is the following.

THEOREM 4.1. Let $f$ be an m-dimensional torus map, and let $A$ be a matrix associated to $f$. If $A$ has an eigenvalue of modulus greater than 1 , then $\operatorname{Per}(f)$ is cofinite in $T_{A}$; that is, there is an $n_{0} \in \mathbf{N}$ such that $\operatorname{Per}(f) \supset T_{A} \backslash\left\{1, \ldots, n_{0}\right\}$.

Proof. We divide the set $E$ of all eigenvalues of $A$ into four subsets $G_{j}$, for $j=1,2,3,4$, by setting

$$
\begin{aligned}
& G_{1}=\{\lambda \in E:|\lambda|>1\}, \\
& G_{2}=\{\lambda \in E:|\lambda|<1\}, \\
& G_{3}=\{\lambda \in E:|\lambda|=1 \text { and } \lambda \text { is not a root of unity }\} \\
& G_{4}=\{\lambda \in E: \lambda \text { is a root of unity }\}
\end{aligned}
$$

The cardinal of $G_{j}$ will be denoted by $k_{j}$ for $j=1,2,3,4$.

Then we have

$$
N\left(A^{n}\right)=\prod_{j=1}^{4} \prod_{\lambda \in G_{j}}\left|1-\lambda^{n}\right| .
$$

We shall study separately the four factors of this equality. We begin with the first factor.

We denote by $\rho$ a real number such that $0<\rho<1,|\lambda|<\rho$ if $\lambda \in G_{2}$, and $|\lambda|^{-1}<\rho$ if $\lambda \in G_{1}$. There exists $n_{1} \in \mathbf{N}$ such that $\rho^{n}<1 / 2$ if $n>n_{1}$. Thus for $n>n_{1}$ we get

$$
\prod_{\lambda \in G_{1}}\left|1-\lambda^{n}\right|=\prod_{\lambda \in G_{1}}|\lambda|^{n}\left|1-\frac{1}{\lambda^{n}}\right|>\frac{1}{2^{k_{1}}} \prod_{\lambda \in G_{1}}|\lambda|^{n}=\frac{1}{2^{k_{1}}} M^{n},
$$

where $M=\prod_{\lambda \in G_{1}}|\lambda|>1$ (in fact, $M$ is the Mahler size of the characteristic polynomial of $A$ ). On the other hand, for each $n \in \mathbf{N}$ we have

$$
\prod_{\lambda \in G_{1}}\left|1-\lambda^{n}\right|=\prod_{\lambda \in G_{1}}|\lambda|^{n}\left|1-\frac{1}{\lambda^{n}}\right|<2^{k_{1}} M^{n}
$$


In a similar way for the second factor we obtain

$$
\frac{1}{2^{k_{2}}}<\prod_{\lambda \in G_{2}}\left|1-\lambda^{n}\right|<2^{k_{2}} \quad \text { if } n>n_{2} .
$$

Now we bound the third factor. Since $A$ is a $m \times m$ matrix with integer entries, its eigenvalues are algebraic numbers of degree less than or equal to $m$. On the other hand, each $n \in \mathbb{N} \backslash\{1\}$ is an algebraic number of degree 1 and height $n$. Suppose now that $\lambda \in G_{3}$. Since $n \log \lambda \neq 0$, applying Theorem 3.1 of [2] we have

$$
\left|\log \lambda^{n}\right|=|n \log \lambda| \geq \frac{1}{n^{c_{\lambda}}},
$$

where the positive constant $c_{\lambda}$ depends only on $m$ and on the height of $\lambda$. If

$$
K=\max \left\{\frac{1}{|z|}: z=t \lambda^{n}+(1-t) \text { with } t \in[0,1]\right\}
$$

then we have

$$
\begin{aligned}
\left|\log \lambda^{n}\right| & =\left|\log \lambda^{n}-\log 1\right|=\left|\log \left(t \lambda^{n}+(1-t)\right)\right|_{t=0}^{t=1} \mid \\
& =\left|\int_{0}^{1} \frac{d}{d t} \log \left(t \lambda^{n}+(1-t)\right) d t\right| \leq K\left|\lambda^{n}-1\right|
\end{aligned}
$$

Therefore

$$
\frac{1}{K n^{c_{\lambda}}} \leq\left|\lambda^{n}-1\right| \text {. }
$$

If $\operatorname{Re} \lambda^{n}>0$ then $K \geq \sqrt{2}$. If $\operatorname{Re} \lambda^{n}<0$ then obviously $\left|\lambda^{n}-1\right|>1$. So we get

$$
\frac{1}{\sqrt{2} n^{c_{\lambda}}} \leq\left|\lambda^{n}-1\right|
$$

if $n$ is sufficiently large, say $n>n_{3}(\lambda)$. Hence

$$
\prod_{\lambda \in G_{3}}\left|1-\lambda^{n}\right| \geq\left(\frac{1}{\sqrt{2}}\right)^{k_{3}} \frac{1}{n^{C k_{3}}} \quad \text { if } n>n_{3},
$$

where $C=\max \left\{c_{\lambda}: \lambda \in G_{3}\right\}$ and $n_{3}=\max \left\{n_{3}(\lambda): \lambda \in G_{3}\right\}$. On the other hand, we have the trivial inequality

$$
\prod_{\lambda \in G_{3}}\left|1-\lambda^{n}\right| \leq 2^{k_{3}} \text {. }
$$


Finally we obtain the bounds for the last factor. Since the set $\left\{\lambda^{n}: \lambda \in G_{4}\right.$ and $\left.\lambda^{n} \neq 1\right\}$ takes finitely many values, we have

$$
\eta^{k_{4}} \leq \prod_{\lambda \in G_{4}}\left|1-\lambda^{n}\right| \leq 2^{k_{4}}
$$

for some $\eta>0$. Then putting together the bounds for the four factors we get

$$
\frac{1}{2^{k_{1}+k_{2}}}\left(\frac{1}{\sqrt{2}}\right)^{k_{3}} \eta^{k_{4}} \frac{1}{n^{C k_{3}}} M^{n} \leq N\left(A^{n}\right) \leq 2^{k_{1}+k_{2}+k_{3}+k_{4}} M^{n},
$$

if $n>n_{4}=\max \left\{n_{1}, n_{2}, n_{3}\right\}$ and $n \in T_{A}$. In short

$$
L \frac{1}{n^{r}} M^{n} \leq N\left(A^{n}\right) \leq 2^{m} M^{n}
$$

if $n>n_{4}$ and $n \in T_{A}$, where $L=\frac{1}{2^{k_{1}+k_{2}}}\left(\frac{1}{\sqrt{2}}\right)^{k_{3}} \eta^{k_{4}}$ and $r=C k_{3}$. Notice that, in fact, the above upper bound of $N\left(A^{n}\right)$ holds for every $n \in \mathbf{N}$.

Now, for each $n \in \mathrm{N}$ we shall find an upper bound of $\sum_{\frac{n}{k}}$ prime $N\left(A^{k}\right)$. Note that for each $k$ such that $n / k$ is prime we have $k \leq \frac{n}{2}$. Then

$$
\sum_{\frac{n}{k} \text { prime }} N\left(A^{k}\right) \leq \sum_{\frac{n}{k} \text { prime }} 2^{m} M^{k} \leq n 2^{m} M^{\frac{n}{2}} .
$$

Since

$$
\lim _{n \rightarrow \infty} \frac{M^{n / 2}}{n^{r+1}}=\infty
$$

there exists $n_{0}>n_{4}$ such that

$$
\frac{M^{n / 2}}{n^{r+1}}>\frac{2^{m}}{L},
$$

if $n \geq n_{0}$. Hence,

$$
L \frac{1}{n^{r}} M^{n}=n 2^{m} M^{n / 2} \frac{L}{2^{m}} \frac{M^{n / 2}}{n^{r+1}}>n 2^{m} M^{n / 2}
$$

for each $n \geq n_{0}$. In short, we have

$$
N\left(A^{n}\right) \geq L \frac{1}{n^{r}} M^{n}>n 2^{m} M^{n / 2} \geq \sum_{\frac{n}{k} \text { prime }} N\left(A^{k}\right),
$$


for each $n \geq n_{0}$ and $n \in T_{A}$. Then, by Proposition 3.4, Corollary 3.3 and Proposition 2.2 the theorem follows.

Corollary 4.2. Let $f$ be an $m$-dimensional torus map, and let $A$ be a matrix associated to $f$. If $A$ has no eigenvalue which is a root of unity and has some eigenvalue different from zero, then $\operatorname{Per}(f)$ is cofinite in $\mathbf{N}$.

Proof. Let $k$ be the multiplicity of the eigenvalue 0 of $A$. Then the characteristic polynomial of $A$ is of the form $\lambda^{k}\left(\lambda^{m-k}+a_{m-k-1} \lambda^{m-k-1}\right.$ $\left.+\ldots .+a_{0}\right)$ with $a_{0} \neq 0$ (because $A$ has an eigenvalue different from zero). Since $A$ is a matrix of integers, $a_{m-k-1}, \ldots, a_{0}$ are integers. Let $\lambda_{1}, \ldots, \lambda_{m-k}$ be the eigenvalues of $A$ different from zero. Then $\left|a_{0}\right|=\left|\lambda_{1}\right| \cdot \ldots \cdot\left|\lambda_{m-k}\right|$.

Suppose that $\left|\lambda_{i}\right| \leq 1$ for $i=1, \ldots, m-k$. Then $\left|a_{0}\right|=1$ and $\left|\lambda_{i}\right|=1$ for $i=1, \ldots, m-k$. It is known $[\mathbf{1 0}]$ that if all the roots of a monic polynomial with integer coefficients have unit modulus, then they all are roots of unity, which contradicts the hypotheses. Thus, $A$ has an eigenvalue of modulus greater than 1 , and the corollary follows from Theorem 4.1 and the fact that $T_{A}=\mathbb{N}$.

By using the same techniques as in the proofs of Theorem 4.1 and Corollary 4.2 we have the following

Corollary 4.3. Let $f$ be an m-dimensional torus map, and let $A$ be a matrix associated to $f$. Then one of the following must occur:

(1) All eigenvalues of A equal zero or a root of unity and, hence, the sequence of Nielsen numbers $\left\{N\left(f^{k}\right)\right\}_{k \geq 1}$ is periodic.

(2) The set $\operatorname{Per}(f)$ contains a cofinite subset of $T_{A}$.

Except for the trivial case when 1 is an eigenvalue of $A$, exactly one of the above statements occurs.

REMARK 4.4. Assume that the hypotheses of Theorem 4.1 are fulfilled. From the proof of this theorem we have $L M^{n} / n^{r} \leq N\left(A^{n}\right)$ for $n \in T_{A}$ sufficiently large. Therefore $\lim _{n \rightarrow \infty} N\left(A^{n}\right)=\infty$. Hence Theorem 4.1 improves Theorem 1 of [6].

In view of Theorem 4.1 a natural question is if there exists a lower bound for $n_{0}$ independent on the dimension of the torus. The negative answer is given by the following proposition. 
Proposition 4.5. Let $m>1$ be any integer, and suppose $A$ is an $m \times m$ matrix of integers having $p(x)=x^{m}+2 x^{m-1}+2 x^{m-2}+$ $\cdots+2 x+2$ as its characteristic polynomial. Then $\operatorname{Per}\left(f_{A}\right)$ is cofinite in $\mathbb{N} \backslash\{k: k>1$ and $k \mid(m+1)\}$.

Proof. Notice that $(x-1) p(x)=x^{m+1}+x^{m}-2$. So, if $z$ is a root of $p(x)$, then $z^{m+1}+z^{m}=2$. Clearly, if $|z|=1$ then $z=1$. But 1 is not a root of $p(x)$. Thus, $p(x)$ has no zeros of modulus 1 . On the other hand $p(x)$ has a root with modulus larger than one (because the product of all roots of $p(x)$ is 2). By Theorem 4.1 it follows that $\operatorname{Per}\left(f_{A}\right)$ is cofinite in $\mathbb{N}$. Now we shall prove that $m+1 \notin \operatorname{Per}\left(f_{A}\right)$. Let $\lambda_{i}$ with $i=1,2, \ldots, m$ be all roots of $p(x)$. Thus,

$$
\begin{aligned}
& \frac{N\left(A^{m+1}\right)}{N(A)}=\prod_{i=1}^{m}\left|\frac{1-\lambda_{i}^{m+1}}{1-\lambda_{i}}\right|=\prod_{i=1}^{m}\left|\lambda_{i}^{m}+\lambda_{i}^{m-1}+\cdots+1\right| \\
& =\prod_{i=1}^{m}\left|\frac{1}{2} \lambda_{i}^{m}+\frac{1}{2}\left(\lambda_{i}^{m}+2 \lambda_{i}^{m-1}+\cdots+2\right)\right|=\prod_{i=1}^{m}\left|\frac{1}{2} \lambda_{i}^{m}\right| \\
& =\frac{1}{2^{m}}\left|\prod_{i=1}^{m} \lambda_{i}\right|^{m}=\left|\frac{\operatorname{det} A}{2}\right|^{m} .
\end{aligned}
$$

Since $\operatorname{det} A=2$, we have $N\left(A^{m+1}\right)=N(A)$. Then the proposition follows from Corollary 3.3.

Now we shall present some sufficient conditions in order that a torus map has periodic points of all periods.

THEOREM 4.6. Let $f$ be an $m$-dimensional torus map and let $A$ be a matrix associated to $f$. Suppose that all the eigenvalues of $A$ are real, positive, different from 1 and $\operatorname{det} A>1$. Then $\operatorname{Per}(f)=\mathbb{N}$.

To prove the theorem we need a lemma.

Lemma 4.7. Let $a \in \mathbb{R}$ with $|a| \neq 1$ and $n \in \mathbb{N}$. Then

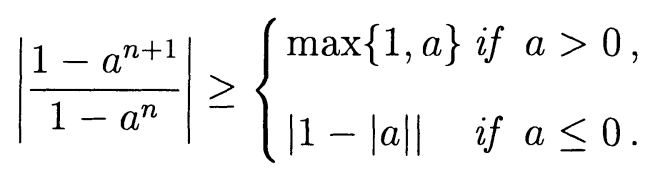


Proof. Suppose $a>0$. Then

$$
\begin{aligned}
& \left|\frac{1-a^{n+1}}{1-a^{n}}\right|=\frac{1+a+\cdots+a^{n}}{1+a+\cdots+a^{n-1}} \\
& \geq \max \left\{\frac{a+\cdots+a^{n}}{1+a+\cdots+a^{n-1}}, \frac{1+a+\cdots+a^{n-1}}{1+a+\cdots+a^{n-1}}\right\}=\max \{a, 1\} .
\end{aligned}
$$

Suppose now $-1<a<0$. If $n$ is even, then

$$
\left|\frac{1-a^{n+1}}{1-a^{n}}\right|=\frac{1+|a|^{n+1}}{1-|a|} \geq 1 \geq 1-|a| \text {. }
$$

If $n$ is odd, then

$$
\begin{aligned}
\left|\frac{1-a^{n+1}}{1-a^{n}}\right| & =\frac{1-|a|^{n+1}}{1+|a|^{n}}=1-\frac{|a|^{n}+|a|^{n+1}}{1+|a|^{n}} \\
& \geq 1-\frac{|a|+|a|^{n+1}}{1+|a|^{n}}=1-|a| .
\end{aligned}
$$

Finally, suppose that $a<-1$. If $n$ is even, then

$$
\left|\frac{1-a^{n+1}}{1-a^{n}}\right|=\frac{|a|^{n+1}+1}{|a|^{n}-1} \geq|a| \geq|a|-1 .
$$

If $n$ is odd, then

$$
\left|\frac{1-a^{n+1}}{1-a^{n}}\right|=\frac{|a|^{n+1}-1}{|a|^{n}+1}=\frac{(|a|-1)\left(|a|^{n}+\cdots+|a|+1\right)}{|a|^{n}+1} \geq|a|-1 \text {. }
$$

Proof of Theorem 4.6. Let $\lambda_{1}, \lambda_{2}, \ldots, \lambda_{m}$ denote all the eigenvalues of $A$. By virtue of Lemma 4.7, we have

$$
\frac{N\left(f^{n+1}\right)}{N\left(f^{n}\right)}=\prod_{i=1}^{m}\left|\frac{1-\lambda_{i}^{n+1}}{1-\lambda_{i}^{n}}\right| \geq \prod_{i=1}^{m} \max \left\{\lambda_{i}, 1\right\} \geq \prod_{i=1}^{m} \lambda_{i}=\operatorname{det} A \geq 2 .
$$

Since $\kappa<2$, by Proposition 3.4, Corollary 3.3 and Theorem 2.7 we get the desired result. 
THEOREM 4.8. Let $f$ be an $m$-dimensional torus map such that its sequence $\left\{N\left(f^{n}\right)\right\}$ of Nielsen numbers is strictly increasing. Then $\operatorname{Per}(f)=\mathbf{N}$.

To prove the theorem we need two lemmas.

LEMma 4.9. Suppose $f: \mathbb{T}^{m} \rightarrow \mathbb{T}^{m}$ is a group homomorphism. If $f$ has periodic points of relatively prime periods $k$ and $j$, then $f$ has a point of period $k j$.

Proof. Let $x$ and $y$ be points of period $k$ and $j$, respectively. Let $z=x \star y$ where $\star$ denotes the group operation on $\mathbb{T}^{m}$. Clearly, $f^{k j}(z)=z$. Therefore, $z$ is a periodic point of $f$ of period $r$ which is a divisor of $k j$. Thus, either $r=k j$ and we are done or there is a prime $p$ such that $p$ divides $k j$ and $r$ divides $k j / p$. Since $k$ and $j$ are relatively prime, $p$ divides at most one of them. So either $k$ or $j$ divides $k j / p$ but not both. Suppose $j$ divides $k j / p$. Then,

$$
x \star y=z=f^{k j / p}(z)=f^{k j / p}(x) \star f^{k j / p}(y)=f^{k j / p(\bmod k)}(x) \star y .
$$

Hence, $f^{k j / p(\bmod k)}(x)=x$, which is a contradiction because $k$ does not divide $k j / p$.

LEMMA 4.10. Let $f$ be an $m$-dimensional torus map such that $N\left(f^{p^{k}}\right)<N\left(f^{p^{k+1}}\right)$ for each prime number $p$ and for each $k \geq 0$. Then $f$ has periodic points of period $p^{k}$ for all $k \geq 1$.

Proof. This follows easily from Proposition 3.4, Corollary 3.3 and Proposition 2.2.

Proof of Theorem 4.8. Let $A$ be the matrix associated to $f$. The map $f_{A}: \mathbb{T}^{m} \rightarrow \mathbb{T}^{m}$ is a group homomorphism. In view of Lemma 4.10 we conclude that $f_{A}$ has periodic points of period $p^{k}$ for each prime $p$ and each $k \geq 1$. Let $n$ be a natural number. We write $n=p_{1}^{k_{1}} p_{2}^{k_{2}} \cdots p_{s}^{k_{s}}$ where $p_{1}, p_{2}, \ldots, p_{s}$ are distinct primes and $k_{i} \geq 1$ for $i=1,2, \ldots, s$. By the inductive use of Lemma 4.9 we get $n \in \operatorname{Per}\left(f_{A}\right)$ which implies $n \in \operatorname{Per}(f)$ by virtue of Proposition 3.4.

REMARK 4.11. From the proof of Theorem 4.8 we see that strict monotonicity is only required in the subsequences of the form $\left\{N\left(f^{p k}\right)\right\}$ for each prime $p$. 
Let $A$ be an $m \times m$ matrix and let $E$ be the set of all eigenvalues of $A$ (each of them repeated according to its multiplicity). Denote by

$$
\begin{aligned}
& G_{1}=\{\lambda \in E: \lambda \in \mathbb{R} \text { and } \lambda>1\}, \\
& G_{2}=\{\lambda \in E: \lambda \in \mathbb{R} \text { and } \lambda<0\}, \\
& G_{3}=\{\lambda \in E: \lambda \in \mathbb{R} \text { and } 0 \leq \lambda<1\}, \text { and } \\
& G_{4}=\{\lambda \in E: \operatorname{Im} \lambda \neq 0\} .
\end{aligned}
$$

Define

$$
\Lambda_{A}=\left(\prod_{\lambda \in G_{1}} \lambda\right) \cdot\left(\prod_{\lambda \in G_{2}}|1-| \lambda||\right) \cdot\left(\prod_{\lambda \in G_{4}}|1-| \lambda||\right)
$$

Then we have:

THEOREM 4.12. Let $f$ be an $m$-dimensional torus map and let $A$ be a matrix associated to $f$. If $A$ has no eigenvalues of modulus 1 and $\Lambda_{A}>1$ then $\operatorname{Per}(f)=\mathbb{N}$.

Proof. As in the proof of Theorem 4.6 we shall look for a lower bound for $N\left(f^{n+1}\right) / N\left(f^{n}\right)$ for all $n \in \mathbb{N}$. We have

$$
\frac{N\left(f^{n+1}\right)}{N\left(f^{n}\right)}=\prod_{\lambda \in E}\left|\frac{1-\lambda^{n+1}}{1-\lambda^{n}}\right|=\prod_{j=1}^{4}\left(\prod_{\lambda \in G_{j}}\left|\frac{1-\lambda^{n+1}}{1-\lambda^{n}}\right|\right) .
$$

Now we are going to estimate from below each factor in the brackets. By Lemma 4.7 we obtain

$$
\begin{aligned}
& \prod_{\lambda \in G_{1}}\left|\frac{1-\lambda^{n+1}}{1-\lambda^{n}}\right| \geq \prod_{\lambda \in G_{1}} \lambda, \\
& \prod_{\lambda \in G_{2}}\left|\frac{1-\lambda^{n+1}}{1-\lambda^{n}}\right| \geq \prod_{\lambda \in G_{2}}|1-| \lambda||, \text { and } \\
& \prod_{\lambda \in G_{3}}\left|\frac{1-\lambda^{n+1}}{1-\lambda^{n}}\right| \geq 1 .
\end{aligned}
$$


Let $\lambda \in G_{4}$ and set $\lambda=r e^{i \theta}$. Then,

$$
\begin{aligned}
\left|\frac{\left(1-\lambda^{n+1}\right)\left(1-\bar{\lambda}^{n+1}\right)}{\left(1-\lambda^{n}\right)\left(1-\bar{\lambda}^{n}\right)}\right| & =\left|\frac{1-2 r^{n+1} \cos (n+1) \theta+r^{2(n+1)}}{1-2 r^{n} \cos n \theta+r^{2 n}}\right| \\
& \geq\left(\frac{1-r^{n+1}}{1+r^{n}}\right)^{2} \geq|1-r|^{2},
\end{aligned}
$$

because

$$
\left|\frac{1-r^{n+1}}{1+r^{n}}\right| \geq|1-r|
$$

Thus,

$$
\prod_{\lambda \in G_{4}}\left|\frac{1-\lambda^{n+1}}{1-\lambda^{n}}\right| \geq \prod_{\lambda \in G_{4}}|1-| \lambda||
$$

Putting together the above inequalities we get

$$
\frac{N\left(f^{n+1}\right)}{N\left(f^{n}\right)} \geq\left(\prod_{\lambda \in G_{1}} \lambda\right) \cdot\left(\prod_{\lambda \in G_{2}}|1-| \lambda||\right) \cdot\left(\prod_{\lambda \in G_{4}}|1-| \lambda||\right)=\Lambda_{A}>1 .
$$

Thus, the theorem follows from Proposition 3.4, Corollary 3.3 and Theorem 4.8.

Next, we prove a result which, in many cases, gives an effective algorithm for computing the minimal set of periods of a torus map.

Proposition 4.13. Let $f$ be an m-dimensional torus map, let $n \in \mathbb{N}$ and $S \subset \mathbb{N}$ be such that any proper divisor of $n$ is also a divisor of some element of $S$. Assume in addition that $N\left(f^{k}\right)>0$ for each $k \in S$. Suppose

$$
N\left(f^{n}\right)>\sum_{k \in S} N\left(f^{k}\right)
$$

Then $f$ has a periodic point of period $n$.

Proof. Since $N\left(f^{k}\right)>0$ for each $k \in S$, from Corollary 3.3 it follows that each essential class of $f_{A}^{j}$ with $j$ a proper divisor of $n$ is contained in an essential class of $f_{A}^{k}$ for some $k \in S$. Since

$$
N\left(f_{A}^{n}\right)=N\left(f^{n}\right)>\sum_{k \in S} N\left(f^{k}\right)=\sum_{k \in S} N\left(f_{A}^{k}\right)
$$


by Corollary 3.3, there is an essential class of $f_{A}^{n}$ which does not contain any essential class of $f_{A}^{j}$ with $j$ a divisor of $n$. Such an essential class contains periodic points of period $n$ for $f_{A}$. Then the statement follows from Proposition 3.4.

THEOREM 4.14. Suppose that the ratio $N\left(f^{n+1}\right) / N\left(f^{n}\right)$ is welldefined and greater than or equal to $\tau$ for all $n \geq n_{0}$ and for some $1<\tau<2$. Then $\operatorname{Per}(f)$ is cofinite in $\mathbf{N}$, in fact, $f$ has periodic points of all periods greater than or equal to the maximum of $2 n_{0}$ and $4\left(1+\left|\frac{\log (\tau-1)}{\log \tau}\right|\right)$.

Proof. Suppose $k \geq \max \left\{2 n_{0}, 4\left(1+\left|\frac{\log (\tau-1)}{\log \tau}\right|\right)\right\}$. Let $j=1+\left[\frac{3 k}{4}\right]$ (we recall that $[x]$ denotes the integer part of $x \in \mathbb{R}$ ). Then since $k \geq 2 n_{0}$, every proper divisor of $k$ is also a divisor of one of the numbers $n_{0}, n_{0}+1, \ldots, j-1$. Since $k-j \geq\left|\frac{\log (\tau-1)}{\log \tau}\right| \geq \Gamma_{\tau}(j)-j$ we have that $\gamma_{\tau}(j) \leq k$ (here we use the notation from Section 2). So $\tau^{k} \geq \sum_{i=0}^{j-1} \tau^{i}$. Therefore

$$
\begin{aligned}
N\left(f^{k}\right) & \geq \frac{1}{\tau^{k}} \sum_{i=0}^{j-1} \tau^{i} N\left(f^{k}\right)>\frac{1}{\tau^{k}} \sum_{i=n_{0}}^{j-1} \tau^{i} N\left(f^{k}\right) \\
& \geq \frac{1}{\tau^{k}} \sum_{i=n_{0}}^{j-1} \tau^{i}\left(\tau^{k-i} N\left(f^{i}\right)\right)=\sum_{i=n_{0}}^{j-1} N\left(f^{i}\right) .
\end{aligned}
$$

Since $N\left(f^{n}\right)>0$ for all $n \geq n_{0}$, Proposition 4.13 implies that $f$ has a periodic point of period $k$.

Note that, if $A$ has no eigenvalues of modulus 1 , and at least one non-zero eigenvalue, then there will be an $n_{0}$ and $\tau$ satisfying the hypotheses of Theorem 4.14 (see Corollary 4.21), and such $n_{0}$ and $\tau$ can be easily calculated if the dimension is reasonable small. The finitely many Nielsen numbers below $k=\max \left\{2 n_{0}, 4\left(1+\left|\frac{\log (\tau-1)}{\log \tau}\right|\right)\right\}$ can then be calculated directly to see which periods below $k$ are present in $\operatorname{MPer}(f)$.

The following theorem shows that the constant $\kappa$ calculated in Theorem 2.7 is the best possible among all spaces satisfying the index assumption.

THEOREM 4.15. For every real number $\alpha$ with $1<\alpha<\kappa$, there exists $X$, a finite disjoint union of $m$-tori, and a continuous map 
$f: X \rightarrow X$ such that the ratio $N\left(f^{n+1}\right) / N\left(f^{n}\right)$ is well-defined and greater than or equal to $\alpha$ for all $n \geq 1$, but $f$ has no periodic points of period 6 so that $\operatorname{Per}(f) \neq \mathbf{N}$.

Proof. Let $1<\alpha<\kappa$. Pick a rational $\beta$ such that $\alpha<\beta<\kappa$ and $\left(\beta+1-\beta^{4}\right)^{-1}>\kappa$. Notice that since $\kappa+1+\kappa^{4}=0$ and $x+1-x^{4}>0$ for $1<x<\kappa$, the inequality $\left(\beta+1-\beta^{4}\right)^{-1}>\kappa$ can be attained by taking $\beta$ sufficiently close to $\kappa$.

By Proposition 4.5, there is an $m \times m$ matrix $A$ of integers such that it has no eigenvalues of modulus $1, \operatorname{det} A=2$ and $\operatorname{Per}\left(f_{A}\right) \cap$ $\{2,3,4,5,6\}=\emptyset$ (notice that a $61 \times 61$ matrix will suffice, although smaller examples probably exist). For each $n>0$ let $M_{n}=N\left(A^{n}\right)$. Since $\operatorname{det} A=2$ and $A$ has no eigenvalues of modulus 1 , it is not difficult to show that $\lim _{n \rightarrow \infty} \frac{M_{n+1}}{M_{n}}=\mu$ exists and is at least 2. Also, $M_{n}=M_{1}$ for $1 \leq n \leq 6$ because $M_{n}$ is exactly the number of fixed points of $f_{A}^{n}$ (see Proposition 3.2).

Now we define rational numbers $P_{k}$ by induction on $k$ in the following way. Set $P_{1}=P_{6}=0, P_{2}=a \beta^{2}-M_{1}, P_{3}=a \beta^{3}-$ $M_{1}, P_{4}=a \beta^{4}-P_{2}-M_{1}$ and $P_{5}=a \beta^{5}-M_{1}$, where $a=M_{1} /\left(\beta^{3}+\right.$ $\left.\beta^{2}-\beta^{6}\right)$. Suppose now that $j \geq 7$ and that the numbers $P_{k}$ have been defined for $k<j$. We set

$$
P_{j}=\max \left\{0, \beta\left(M_{j-1}+\sum_{k \mid(j-1)} P_{k}\right)-\left(M_{j}+\sum_{\substack{k<j \\ k \mid j}} P_{k}\right)\right\} .
$$

Since $\lim _{n \rightarrow \infty} \frac{M_{n+1}}{M_{n}}=\mu$, then $M_{n}=O\left(\mu^{n}\right)$. Thus, since $\mu \geq 2>\beta$, from the definition of the numbers $P_{j}$ one can show by induction that $P_{j}=O\left(\beta^{j}\right)$. Therefore, there exists $j_{0} \in \mathbf{N}$ such that $P_{j}=0$ for all $j>j_{0}$.

Now, set

$$
N_{j}=M_{j}+\sum_{k \mid j} P_{k}
$$

for all $j \in \mathbf{N}$. We note that $N_{1}=M_{1}, N_{k}=a \beta^{k}$ for $2 \leq k \leq 6$ and that

$$
P_{j}=\max \left\{0, \beta N_{j-1}-\left(N_{j}-P_{j}\right)\right\}
$$

for all $j \geq 7$. Hence, for $j \geq 7$ we have $P_{j} \geq \beta\left(N_{j-1}-\left(N_{j}-P_{j}\right)\right)$ which is equivalent to $N_{j} \geq \beta N_{j-1}$. Therefore, $\frac{N_{j+1}}{N_{j}} \geq \beta$ for $j \geq 2$. 
Furthermore,

$$
\frac{N_{2}}{N_{1}}=a \frac{\beta^{2}}{M_{1}}=\left(\beta+1-\beta^{4}\right)^{-1}>\kappa>\beta .
$$

Let $r$ be the smallest positive integer such that $r P_{j} \in \mathbb{Z}$ for all $j \in \mathbb{N}$. Note that such an $r$ exists because $P_{j}=0$ for $j>j_{0}$ and $P_{j} \in \mathbb{Q}$ for $j \leq j_{0}$.

Now let $X$ be a disjoint union of $r \cdot j_{0} ! \cdot\left(1+P_{2}+P_{3}+\cdots+P_{j_{0}}\right)$ copies of $\mathbb{T}^{m}$. We define the map $f: X \rightarrow X$ as follows. We

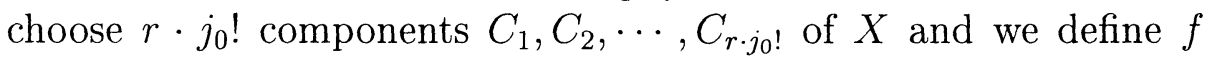
on these components such that $f\left(C_{i}\right)=C_{i}$ and $\left.f\right|_{C_{i}}=\left.f_{A}\right|_{C_{i}}$ for $i=1,2, \cdots, r \cdot j_{0}$ !. Now let us fix $k \in\left\{2,3, \ldots, j_{0}\right\}$. Notice that $r \cdot j_{0} ! \cdot P_{k} \in \mathbb{N}$ and $k$ divides $r \cdot j_{0} ! \cdot P_{k}$. Then we take $\left(r \cdot j_{0} ! \cdot P_{k}\right) / k$ groups of $k$ connected components of $X$. On each of these groups we define $f$ in the following way. Let $K_{1}, K_{2}, \ldots, K_{k}$ be the connected components of one of these groups. We set $f\left(K_{i}\right)=K_{i+1}$ for $i=$ $1,2, \ldots, k-1$ and $f\left(K_{k}\right)=K_{1}$ in such a way that $\left.f\right|_{K_{i}}$ is constant for each $i$.

In that way, each group of $k$ components gives a unique periodic orbit which has period $k$. This ends the construction of the map $f$. We note that:

(1) Each periodic point of period $k$ of $f$ forms an essential class of $f^{k}$ (see Proposition 3.2).

(2) $N\left(\left.f^{k}\right|_{C_{1} \cup C_{2} \cup \cdots \cup C_{r \cdot J_{0} !}}\right)=r \cdot j_{0} ! \cdot N\left(f_{A}^{k}\right)=r \cdot j_{0} ! \cdot M_{k}$ for all $k \in \mathbb{N}$.

(3) $N\left(f^{k} \mid X \backslash\left\{C_{1} \cup C_{2} \cup \cdots \cup C_{r \cdot j_{0} !}\right\}\right)=\sum_{\substack{j \mid k \\ j \leq j_{0}}} r \cdot j_{0} ! \cdot P_{j}$.

Therefore, since $P_{j}=0$ for $j \geq j_{0}$, in view of $(*)$ we get that $N\left(f^{k}\right)=r \cdot j_{0} ! \cdot N_{k}$ for all $k \in \mathbb{N}$. Hence, $N\left(f^{k+1}\right) / N\left(f^{k}\right) \geq \beta$ for all $k$. On the other hand, the number of periodic points of period 6 of $f$ is $N\left(f^{6}\right)-N\left(f^{3}\right)-N\left(f^{2}\right)+N(f)$. By straightforward computation this number turns out to be zero. So $f$ has no periodic points of period 6 .

REMARK 4.16. We note that the map constructed in Theorem 4.15 depends on moving around the very numerous components of $X$. So, if $1<\alpha<\kappa$, does there exist a compact connected 
manifold $X$ and a continuous map $f: X \rightarrow X$ such that the ratio $N\left(f^{n+1}\right) / N\left(f^{n}\right)$ is well-defined and greater than or equal to $\alpha$ for all $n \geq 1$, yet $\operatorname{Per}(f) \neq \mathbb{N}$.

Now we give a complete characterization of all the sets $\operatorname{MPer}\left(f_{A}\right)$ corresponding to all two-dimensional torus maps.

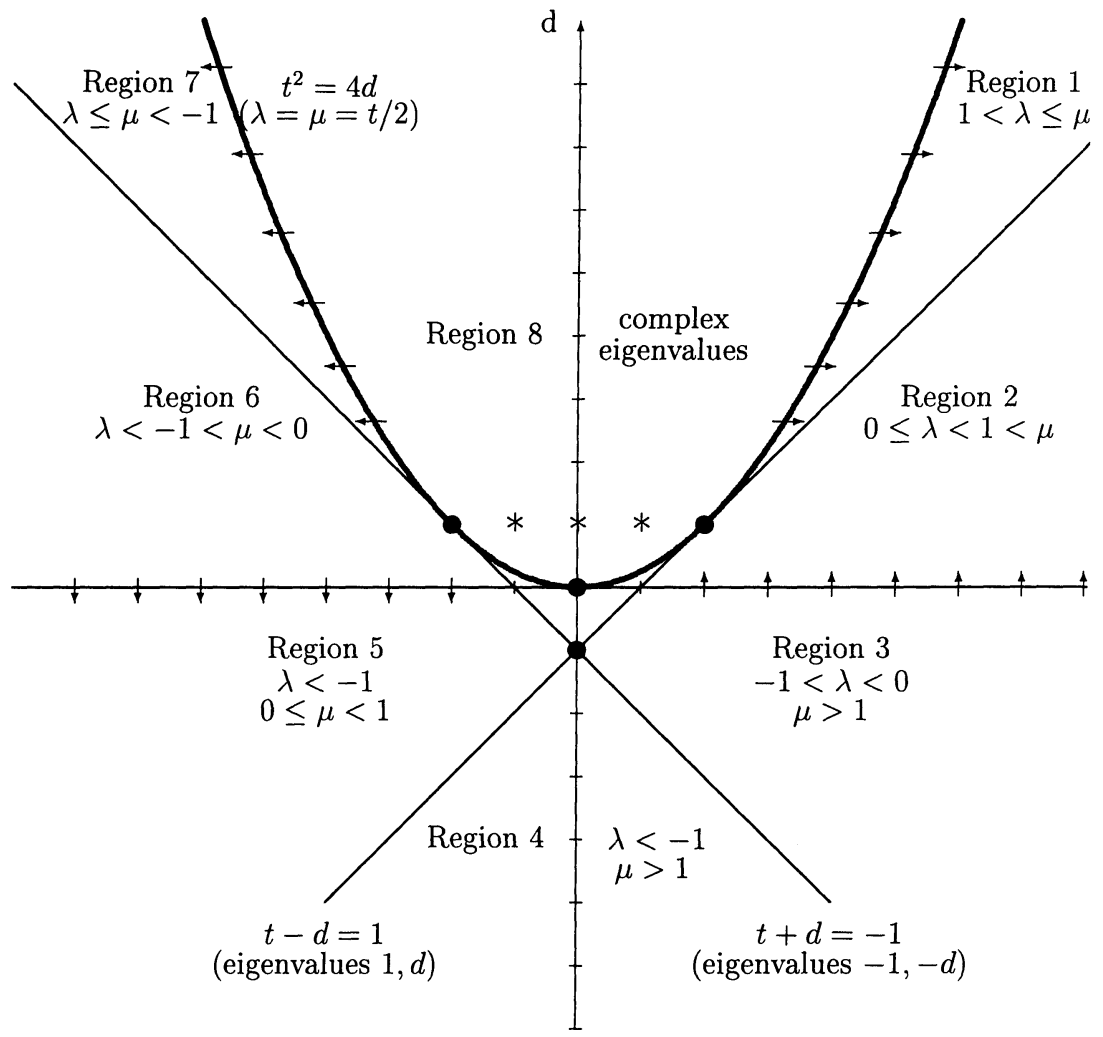

Figure 1. The regions under consideration in the $(t, d)$-plane.

The characterization of the sets $\operatorname{MPer}\left(f_{A}\right)$ will be achieved by studying the sequences $\left\{N\left(A^{n}\right)\right\}$. Matrices having the same pair of eigenvalues have the same sequence $\left\{N\left(A^{n}\right)\right\}$ (and therefore the same set $\left.\operatorname{MPer}\left(f_{A}\right)\right)$. Since in this section $A$ is a $2 \times 2$ matrix, these sequences depend on the two eigenvalue parameters only. A more convenient choice of parameters is the trace and the determinant of the matrix $A$, because they take only integer values.

In the sequel we denote by $t=t(A)$ and $d=d(A)$ the trace and determinant of the matrix $A$, respectively. Then the eigenvalues of 
$A$ are $\lambda=\left(t-\sqrt{t^{2}-4 d}\right) / 2$ and $\mu=\left(t+\sqrt{t^{2}-4 d}\right) / 2$.

If $T_{A}=\emptyset$, i.e. all of the Nielsen numbers $N\left(A^{n}\right)$ are 0 , then 1 is an eigenvalue of $A$. Since $d$ is then the other eigenvalue we have $t=d+1$. Thus on the line $t-d=1$ we have $T_{A}=\emptyset$ and $\operatorname{MPer}\left(f_{A}\right)=\emptyset$. Similarly, if -1 is an eigenvalue, then $t+d=-1$, and we have that $T_{A}$ is the set of positive odd numbers (except where the two lines intersect at $(0,-1))$. In Figure 1 , we see the $(t, d)$-plane divided into several regions, in which the arguments will be somewhat different. The $d$-axis is not the boundary of any region. The $t$-axis (for $|t|>1$ ) and the line $t^{2}=4 d$ (for $|d|>1$ ) are each included in one of the regions which they bound (indicated by small arrows in Figure 1). Thus, for example, the $t$-axis for $|t|>1$ is included in Region 2. The origin $(t=d=0)$ and the points on the lines $t+d=-1$ and $t-d=1$ are not in any region, and will be discussed as separate cases. The two lines $t+d=-1$ and $t-d=1$ and the three points $( \pm 1,1)$ and $(0,1)$ correspond to matrices having roots of unity as eigenvalues, and therefore $T_{A} \neq \mathbf{N}$.

Now we shall analyze the sets $\operatorname{MPer}\left(f_{A}\right)$ for each of the regions in Figure 1.

Proposition 4.17. Suppose $(t, d)$ is not on either of the lines $d+t=-1$ or $t-d=1$. Then $2 \notin \operatorname{MPer}\left(f_{A}\right)$ if and only if $d+t$ is either 0 or -2 .

Proof. Clearly, $N(A) \neq 0$ and $N\left(A^{2}\right) \neq 0$. Then,

$$
\begin{aligned}
\frac{N\left(A^{2}\right)}{N(A)} & =\left|\frac{\left(1-\lambda^{2}\right)\left(1-\mu^{2}\right)}{(1-\lambda)(1-\mu)}\right| \\
& =|(1+\lambda)(1+\mu)| \\
& =|1+\lambda+\mu+\lambda \mu|=|1+t+d| .
\end{aligned}
$$

Thus $N\left(A^{2}\right)=N(A)$ if and only if $t+d$ is either 0 or -2 .

In short, we have two lines which are parallel to the -1 eigenvalue line $t+d=-1$, on which period 2 is missing from the canonical torus $\operatorname{map} f_{A}$.

Our next proposition shows that for the case of real eigenvalues, any values of $t$ and $d$ which do not satisfy one of the above restrictions automatically gives us periodic points of all periods. 
Proposition 4.18. Suppose A has real eigenvalues, neither of which are \pm 1 . If $d+t$ is neither 0 nor -2 , then $\operatorname{MPer}\left(f_{A}\right)=\mathbf{N}$.

Proof. Note that if $A$ satisfies all of the hypotheses of the proposition, then $(t, d)$ is in one of the Regions 1 through 7 in Figure 1.

Since the hypotheses imply that $A$ has no roots of unity as eigenvalues, $N\left(A^{n}\right) \neq 0$ for all $n \geq 1$ (see Lemma 3.1). Thus,

$$
\frac{N\left(A^{n+1}\right)}{N\left(A^{n}\right)}=\left|\frac{\left(1-\lambda^{n+1}\right)\left(1-\mu^{n+1}\right)}{\left(1-\lambda^{n}\right)\left(1-\mu^{n}\right)}\right|
$$

for all $n$. Since $\lambda$ and $\mu$ are real and neither is \pm 1 , we can use Lemma 4.7 (with $a$ replaced by $\lambda$ and $\mu$ ) to get a simple expression for a lower bound $E$ for $\frac{N\left(A^{n+1}\right)}{N\left(A^{n}\right)}$, which will differ depending on the region under consideration. For example, in Region 3, we have $-1<\lambda<0$ and $\mu>1$. Then, $E=(1-|\lambda|)|\mu|$ in that region. Similar expressions can be obtained for the other regions.

Thus, $N\left(A^{n+1}\right) \geq E \cdot N\left(A^{n}\right)$ in each case. By Theorem 4.8, we know that if the sequence $\left\{N\left(A^{n}\right)\right\}$ is strictly increasing, then $\operatorname{MPer}\left(f_{A}\right)=\operatorname{Per}\left(f_{A}\right)=\mathbb{N}$. Thus, it is enough to show that $E>1$. For Regions 1 and 2 this is the case but in Regions 3 through 7, one sometimes has $E \leq 1$ (because of the lines $t+d=0$ and $t+d=-2$; see Proposition 4.17). Then the proposition follows because, in those regions, the assumption $t+d \neq 0,-2$ implies $E>1$.

We now need to examine the special case where $d+t$ is 0 or -2 . Those giving complex eigenvalues will be dealt with later. First, a special and easy case is considered separately.

Proposition 4.19. If $d=t=0$, the $T_{A}=\emptyset$ and $\operatorname{MPer}\left(f_{A}\right)=$ $\{1\}$.

We are left with the values of $(t, d)$ in which $d+t=0,-2$ and $(t, d)$ is in one of the Regions numbered 3 through 7 . Since, by Proposition 4.17, there are no period 2 points for these values, we are left with the possibility of using results such as Theorems $2.7,2.9$ and 2.10. Thus, we would like to find integers $n_{0}$ (which will depend on $t$ and $d$ ) such that $\frac{N\left(A^{n+1}\right)}{N\left(A^{n}\right)}>\kappa$ for $n \geq n_{0}$, (where $\kappa$ is as in Section 2 ), and $n_{0}$ is as small as possible. Since $\kappa \approx 1.22$, we will use $5 / 4$ instead of $\kappa$ for ease on the computations. In general, $\frac{N\left(A^{n+1}\right)}{N\left(A^{n}\right)}$ is 
not monotone, so knowing that $\frac{N\left(A^{n+1}\right)}{N\left(A^{n}\right)}>\kappa$ for a particular value of $n$ does not tell us about larger values. To deal with this, we will find a lower bound estimate for $\frac{N\left(A^{n+1}\right)}{N\left(A^{n}\right)}$ which is monotone with respect to $n$, so that proving this estimate is greater than $\kappa$ for some $n$ automatically gives us the same fact for all larger values of $n$. Thus our basic strategy will be:

(1) Find an estimate $E_{n}$ such that $\frac{N\left(A^{n+1}\right)}{N\left(A^{n}\right)} \geq E_{n}$ for all $n$ (and all suitable $A$ ), and such that $E_{n+1} \geq E_{n}$ for all $n$.

(2) Find an $n_{0}$ such that $E_{n_{0}} \geq 5 / 4$, which will guarantee $\frac{N\left(A^{n+1}\right)}{N\left(A^{n}\right)}$ $>\kappa$ for all $n \geq n_{0}$.

(3) Use Theorems 2.7, 2.9 and 2.10 in order to find a finite set $F$ such that $\operatorname{Per}\left(f_{A}\right) \supset \mathbf{N} \backslash F$. If $n_{0}$ is 1 or 2 , then $\operatorname{MPer}\left(f_{A}\right) \equiv \operatorname{Per}\left(f_{A}\right)$ is either $\mathbf{N}$ or $\mathbf{N} \backslash\{2\}$, and Proposition 4.17 can be used to decide which. If $n_{0} \geq 3$ then we still have a fixed finite set $F$, and a direct calculation of all of the Nielsen numbers up to the largest element of $F$ tells us which periods are or are not there. This must be done separately for each matrix $A$ such that $n_{0} \geq 3$.

If the above strategy is going to work, there is one key thing which must happen. If we try this strategy on a given class of matrices, then we must have $n_{0}=1$ or 2 for all but finitely many pairs $(t, d)$ and have a fixed $n_{0}$ for each of the finitely many remaining ones. Only then will this strategy result in reducing the problem to a finite number of calculations. This can be done so that the total number of calculations is relatively small. In the sequel we will omit the proofs of all the steps towards the characterization of the sets $\operatorname{MPer}\left(f_{A}\right)$ for two-dimensional torus maps. Some of these proofs are omitted because they are straightforward computations and the other ones because they are tedious repetitions of previous arguments. We start with the following technical lemma.

LEMMA 4.20. The following statements hold.

(1) Let $a$ be a complex number such that $|a| \neq 1$. Then for all $n \geq 1$,

$$
\left|\frac{1-a^{n+1}}{1-a^{n}}\right| \geq\left|\frac{1-|a|^{n+1}}{1+|a|^{n}}\right| .
$$

(2) Let $r \geq 0$ be real, $r \neq 1$. Then for all $n \geq 1$,

$$
\left|\frac{1-r^{n+2}}{1+r^{n+1}}\right| \geq\left|\frac{1-r^{n+1}}{1+r^{n}}\right| \text {. }
$$


(3) If a is a complex number such that $|a| \neq 1$, then for all $n \geq k \geq 1$

$$
\left|\frac{1-a^{n+1}}{1-a^{n}}\right| \geq\left|\frac{1-|a|^{k+1}}{1+|a|^{k}}\right| .
$$

Noting the above results, if $B$ is an $m \times m$ matrix with eigenvalues $\lambda_{1}, \ldots, \lambda_{m}$ with $\left|\lambda_{i}\right| \neq 1$ for all $i$, we define

$$
E_{k}(B)=\prod_{i=1}^{m}\left|\frac{1-\left|\lambda_{i}\right|^{k+1}}{1+\left|\lambda_{i}\right|^{k}}\right| .
$$

Then we get:

Corollary 4.21. If $B$ has no eigenvalues having modulus 1, then for all $n \geq k \geq 1$

$$
\frac{N\left(B^{n+1}\right)}{N\left(B^{n}\right)} \geq E_{k}(B) .
$$

Going back to our $2 \times 2$ matrix $A$ described at the beginning of this section, let us abbreviate $E_{k}(A)$ by $E_{k}$.

The following result allows us to compute easily the Nielsen numbers $N\left(A^{n}\right)$ for a $2 \times 2$ matrix $A$. To state it we set $N_{n}=(1-$ $\left.\lambda^{n}\right)\left(1-\mu^{n}\right)$ for $n \geq 0$. We note that, for $n \geq 1$, the Nielsen number $N\left(A^{n}\right)$ is simply the absolute value of $N_{n}$.

Proposition 4.22. We have

$$
N_{n}=t N_{n-1}-d N_{n-2}+N_{1}\left(1+d^{n-1}\right)
$$

for all $n \geq 2$.

Proposition 4.23. Suppose $t+d$ is either 0 or $-2,(t, d) \neq$ $(0,0)$, and $\lambda$ and $\mu$ are real. Then $\operatorname{MPer}\left(f_{A}\right)=\mathbb{N} \backslash\{2\}$.

The following result takes care of the cases in which complex eigenvalues occur, except for a finite number of cases which will be considered later.

Proposition 4.24. If $d \geq 2$ and $\lambda$ and $\mu$ are not real, then $\operatorname{MPer}\left(f_{A}\right)$ is $\mathbb{N}$, unless the pair $(t, d)$ is among the following four exceptions:

(1) If $(t, d)=(-3,2)$, then $\operatorname{MPer}\left(f_{A}\right)=\mathbb{N} \backslash\{2\}$. 
(2) If $(t, d)=(-2,2)$, then $\operatorname{MPer}\left(f_{A}\right)=\mathbf{N} \backslash\{2,3\}$.

(3) If $(t, d)=(-1,2)$, then $\operatorname{MPer}\left(f_{A}\right)=\mathbf{N} \backslash\{3\}$.

(4) If $(t, d)=(0,2)$, then $\operatorname{MPer}\left(f_{A}\right)=\mathbf{N} \backslash\{4\}$.

This takes care of all values of $(t, d)$ in which no roots of unity occur as eigenvalues. We now turn to the roots of unity case.

Proposition 4.25. Suppose $t+d=-1$ (i.e. -1 is an eigenvalue of $A$ ). Then the following hold:

(1) If $d=-1$, then $T_{A}=\emptyset$ and $\operatorname{MPer}\left(f_{A}\right)=\emptyset$.

(2) If $d \in\{0,1\}$, then $T_{A}$ is the set of all odd natural numbers and $\operatorname{MPer}\left(f_{A}\right)=\{1\}$.

(3) If $d \in \mathbb{Z} \backslash\{-1,0,1\}$, then $T_{A}=\operatorname{MPer}\left(f_{A}\right)$ is the set of all odd natural numbers.

All that is left is the three values where $d=1$ and the eigenvalues are complex.

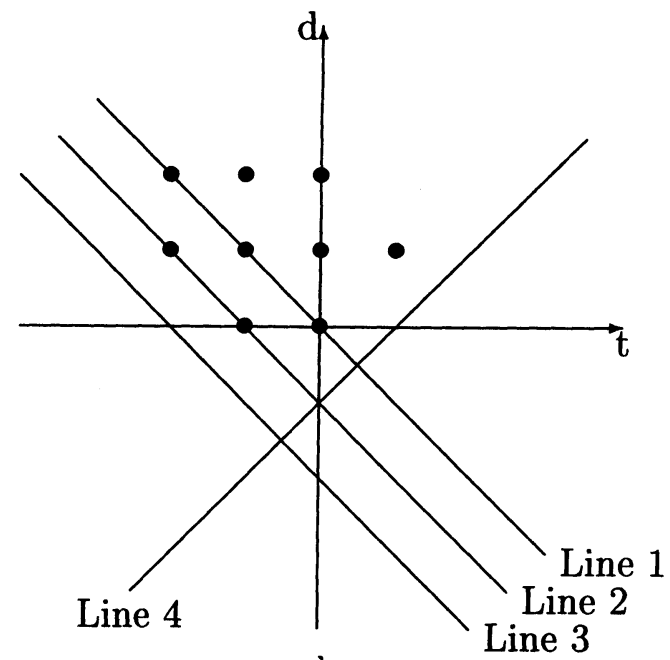

Line 1: $t+d=0 ; \operatorname{MPer}\left(f_{A}\right)=\dot{\mathbf{N}} \backslash\{2\}$.

Line 2: $t+d=-1$, and $t \neq 0 ; \operatorname{MPer}\left(f_{A}\right)=\{n \in \mathbf{N}: n$ is odd $\}$.

Line 3: $t+d=-2 ; \operatorname{MPer}\left(f_{A}\right)=\mathbf{N} \backslash\{2\}$.

Line 4: $t-d=1 ; \operatorname{MPer}\left(f_{A}\right)=\emptyset$.

Figure 2. If $(t, d)$ is off the four special lines $(t+d=$ $0,-1,-2, t-d=1)$ and the nine exceptional cases (marked as $\bullet$ ) then $\operatorname{Per}\left(f_{A}\right)=\operatorname{MPer}\left(f_{A}\right)=\mathbf{N}$. The set $\operatorname{MPer}\left(f_{A}\right)$ for the exceptional cases is given in Table 1. 


\begin{tabular}{|c|c|}
\hline$(t, d)$ & $\operatorname{MPer}\left(f_{A}\right)$ \\
\hline$(-2,1)$ & $\{1\}$ \\
\hline$(-2,2)$ & $\mathbb{N} \backslash\{2,3\}$ \\
\hline$(-1,0)$ & $\{1\}$ \\
\hline$(-1,1)$ & $\{1\}$ \\
\hline$(-1,2)$ & $\mathbb{N} \backslash\{3\}$ \\
\hline$(0,0)$ & $\{1\}$ \\
\hline$(0,1)$ & $\{1,2\}$ \\
\hline$(0,2)$ & $\mathbb{N} \backslash\{4\}$ \\
\hline$(1,1)$ & $\{1,2,3\}$ \\
\hline
\end{tabular}

TABLE 1. The set $\operatorname{MPer}\left(f_{A}\right)$ in the nine exceptional cases.

Proposition 4.26. Suppose $d=1$ and $-1 \leq t \leq 1$. Then

(1) If $t=-1$, then $T_{A}=\{n \in \mathbb{N}: n \not \equiv 0(\bmod 3)\}$ and $\operatorname{MPer}\left(f_{A}\right)=\{1\}$.

(2) If $t=0$, then $T_{A}=\{n \in \mathbb{N}: n \not \equiv 0(\bmod 4)\}$ and $\operatorname{MPer}\left(f_{A}\right)$ $=\{1,2\}$.

(3) If $t=1$, then $T_{A}=\{n \in \mathbb{N}: n \not \equiv 0(\bmod 6)\}$ and $\operatorname{MPer}\left(f_{A}\right)$ $=\{1,2,3\}$.

If we put all the above propositions together, we can summarize the results in Figure 2 and Table 1 . Note that if in this figure and table we set $d=0$, then the above results just reduce to the already known results on the circle (mentioned in the introduction).

Acknowledgements. S. Baldwin and R. Swanson kindly thank the Centre de Recerca Matemàtica for their support while this paper was being written. R. Swanson was also supported, in part, by a grant from National Science Foundation. W. Szlenk thanks the invitation of the Universitat Autònoma de Barcelona. Ll. Alsedà and J. Llibre have been partially supported by the DGICYT grant number PB90-0695. We thank the referee for carefully reading the manuscript and contributing valuable remarks. 


\section{REFERENCES}

[1] Ll. Alsedà, J. Llibre and M. Misiurewicz, Combinatorial dynamics and entropy in dimension one, Advanced Series on Nonlinear Dynamics 5, World Scientific, 1993.

[2] A. Baker, Transcendental number theory, Cambridge Univ. Press, 1975.

[3] L. Block, J. Guckenheimer, M. Misiurewicz and L.S. Young, Periodic points and topological entropy of one dimensional maps, Global Theory of dynamical systems, 18-34, Lecture Notes in Math. 819, Springer, Berlin, 1980.

[4] R.B.S. Brooks, R.F. Brown, J. Pak and D.H. Taylor, Nielsen numbers of maps of tori, Proc. Amer. Math. Soc. 52 (1975), 398-400.

[5] L.S. Efremova, Periodic orbits and the degree of a continuous map of a circle (in Russian), Diff. and Integer Equations (Gor'kii) 2, (1978), 109-115.

[6] B. Halpern, Periodic points on tori, Pacific J. of Math. 83 (1979), 117133.

[7] P. Heath, R. Piccinini and C.Y. You, Nielsen-type numbers for periodic points, I, Lecture Notes in Math. 1411(1988), 88-106.

[8] B. Jiang, Lectures on Nielsen fixed point theory, Contemporary Mathematics 14, Amer. Math. Soc., 1983.

[9] T.H. Kiang, The theory of fixed point classes, Springer-Verlag, Berlin, 1989.

[10] H.E. Leipzig, Theorie der algebraischen zahlen, Akademische Verlagsgesellschaft, 1923.

[11] C. Simó, R. Swanson and R. Walker, Torus maps with fewest periodic orbits, preprint.

Received September 30, 1992 and in revised form June 10, 1993.

Universitat Autonoma de BARCELONA 08193-Bellaterra, Barcelona, Spain

AND

AUbURn UNIVERSITY

AUBURN UNIVERSITY, AL 36849

AND

Montana State University

BOZEMAN, MT 59717

E-mail address: dswanson@math.montana.edu 


\title{
PACIFIC JOURNAL OF MATHEMATICS
}

Founded by E. F. Beckenbach (1906-1982) and F. Wolf (1904-1989)

\section{EDITORS}

Sun-Yung Alice Chang (Managing Editor)

University of California

Los Angeles, CA 90095-1555

pacific@math.ucla.edu

F. Michael Christ

University of California

Los Angeles, CA 90095-1555

christ@math.ucla.edu

Thomas Enright

University of California

San Diego, La Jolla, CA 92093

tenright@ucsd.edu

Nicholas Ercolani

University of Arizona

Tucson, AZ 85721

ercolani@math.arizona.edu
Robert Finn

Stanford University

Stanford, CA 94305

finn@gauss.stanford.edu

Vaughan F. R. Jones

University of California

Berkeley, CA 94720

vfr@math.berkeley.edu

Steven Kerckhoff

Stanford University

Stanford, CA 94305

spk@gauss.stanford.edu
Martin Scharlemann

University of California

Santa Barbara, CA 93106

mgscharl@math.ucsb.edu

\section{Gang Tian}

Courant Institute

New York University

New York, NY 10012-1100

tiang@taotao.cims.nyu.edu

V. S. Varadarajan

University of California

Los Angeles, CA 90095-1555

vsv@math.ucla.edu

\section{SUPPORTING INSTITUTIONS}

\section{CALIFORNIA INSTITUTE OF TECHNOLOGY \\ NEW MEXICO STATE UNIVERSITY \\ OREGON STATE UNIVERSITY \\ STANFORD UNIVERSITY \\ UNIVERSITY OF ARIZONA \\ UNIVERSITY OF BRITISH COLUMBIA \\ UNIVERSITY OF CALIFORNIA UNIVERSITY OF HAWAII}

\author{
UNIVERSITY OF MONTANA \\ UNIVERSITY OF NEVADA, RENO \\ UNIVERSITY OF OREGON \\ UNIVERSITY OF SOUTHERN CALIFORNIA \\ UNIVERSITY OF UTAH \\ UNIVERSITY OF WASHINGTON \\ WASHINGTON STATE UNIVERSITY
}

The supporting Institutions listed above contribute to the cost of publication of this Journal, but they are not owners or publishers and have no responsibility for its contents or policies.

Manuscripts must be prepared in accordance with the instructions provided on the inside back cover.

The Pacific Journal of Mathematics (ISSN 0030-8730) is published monthly except for July and August. Regular subscription rate: $\$ 215.00$ a year (10 issues). Special rate: $\$ 108.00$ a year to individual members of supporting institutions.

Subscriptions, orders for back issues published within the last three years, and changes of subscribers address should be sent to Pacific Journal of Mathematics, P.O. Box 4163, Berkeley, CA 94704-0163, U.S.A. Prior back issues are obtainable from Kraus Periodicals Co., Route 100, Millwood, NY 10546.

The Pacific Journal of Mathematics at the University of California, c/o Department of Mathematics, 981 Evans Hall, Berkeley, CA 94720 (ISSN 0030-8730) is published monthly except for July and August. Second-class postage paid at Berkeley, CA 94704, and additional mailing offices. POSTMASTER: send address changes to Pacific Journal of Mathematics, P.O. Box 6143, Berkeley, CA 94704-0163.

\author{
PUBLISHED BY PACIFIC JOURNAL OF MATHEMATICS at University of California, \\ Berkeley, CA 94720, A NON-PROFIT CORPORATION \\ This publication was typeset using AMS-LATEX, \\ the American Mathematical Society's TEX macro system. \\ Copyright (C) 1995 by Pacific Journal of Mathematics
}




\section{PACIFIC JOURNAL OF MATHEMATICS}

Volume 169 No. $1 \quad$ May 1995

Minimal sets of periods for torus maps via Nielsen numbers

Lluís AlsedÀ, StewART BALdWin, JAUME Llibre,

RICHARD SWANSON and WIESLAW SZLENK

Diagonalizing Hilbert cusp forms

Timothy ATWILL

A splitting criterion for rank 2 vector bundles on $\mathbf{P}^{n}$

EDOARDO BALLICO

Controlling Tietze-Urysohn extensions

MARC FRANTZ

Length of Julia curves

DAVID H. HAMILTON

On the uniqueness of capillary surfaces over an infinite strip

JENN-FANG HWANG

Volume estimates for log-concave densities with application to iterated 107 convolutions

MARIUS JUNGE

A reflection principle in complex space for a class of hypersurfaces and mappings

FRANCINE ANTOINETTE MEYLAN

Jean Bourgain's analytic partition of unity via holomorphic martingales 161

PAUL F.X. MÜLLER

Characters of Brauer's centralizer algebras 